\title{
Fabrication and testing of freestanding Si nanogratings for UV filtration on space-based particle sensors
}

\author{
Pran Mukherjee ${ }^{1}$, Thomas H Zurbuchen and L Jay Guo \\ The University of Michigan, 2417A SRB, 2455 Hayward Street, Ann Arbor, \\ MI 48109-2143, USA \\ E-mail: pran@umich.edu
}

Received 9 April 2009, in final form 22 June 2009

Published 21 July 2009

Online at stacks.iop.org/Nano/20/325301

\begin{abstract}
We demonstrate complete fabrication process integration and device performance of sturdy, self-supported transmission gratings in silicon. Gratings are patterned with nanoimprint lithography and aluminum liftoff on silicon-on-insulator wafers. Double-sided deep reactive ion etching (DRIE) creates freestanding $120 \mathrm{~nm}$ half-pitch gratings with $2000 \mathrm{~nm}$ depth and built-in $1 \mathrm{~mm}$ pitch bulk silicon support structures. Optical characterization demonstrates $10^{-4}$ transmission of UV in the 190-250 nm band while a 25-30\% geometric transparency allows particles to pass unimpeded for space plasma measurements.
\end{abstract}

(Some figures in this article are in colour only in the electronic version)

\section{Introduction}

Satellite missions designed for space weather monitoring and forecasting often carry instruments for measuring the composition of plasmas and neutral atom populations in space. The Sun's continuous, omnidirectional particle stream-the solar wind-is heated and accelerated in the Sun's outer atmosphere by mechanisms that are so far not well understood. Detailed in situ measurements of the solar wind are essential for understanding of these energy transfer mechanisms.

Instruments sensitive enough to measure the individual atom or ion (henceforth: particle) impacts of low-density plasmas like the solar wind are often highly vulnerable to energetic ultraviolet photons, which can degrade the instrument performance or produce noise in the system. In particular, the Lyman- $\alpha$ band of the UV at $121.6 \mathrm{~nm}$ $(10.25 \mathrm{eV})$, caused by excited hydrogen emission in the Sun, can produce a signal that is indistinguishable from the solar wind particle signal $[1,2]$. Thus, a filter that blocks the energetic UV photons while allowing particles through for measurement is required.

For space-based particle sensors the best stand-alone UV filters to date are perforated foils or gratings. They work on the

1 Present address: Massachusetts Institute of Technology, Room 37-415, 77 Massachusetts Avenue, Cambridge, MA 02139-4307, USA. principle that photons can only pass through a straight channel if the channel width is much larger than the photon wavelength, which is on the order of tens or hundreds of nanometers. In contrast, atomic widths are on the order of angstroms, with de Broglie wavelengths ${ }^{2}$ measured in picometers [3]. For example, the de Broglie wavelength of slow solar wind protons $\left(450 \mathrm{~km} \mathrm{~s}^{-1}\right)$ is $8.8 \times 10^{-13} \mathrm{~m}$, or just under $1 \mathrm{pm}$.

In order to be an effective UV filter, a grating must have a large geometric transparency to pass particles and very narrow, high aspect ratio slits to block energetic photons [4-6]. Such nanoscale transmission gratings have already been flown on a variety of space-based sensor and instrument applications. Ultraviolet filtration on the medium energy neutral atom (MENA) instrument for the IMAGE satellite was accomplished by gold gratings with $40 \mathrm{~nm}$ slits and $510 \mathrm{~nm}$ thickness [7-9]. Similar gratings were used in experiments with extreme ultraviolet (EUV) diffraction [10] and $0.5 \mathrm{keV}$ electron diffraction $[3,11]$. High resolution x-ray spectroscopy on NASA's Chandra space telescope was enabled by 200 and $400 \mathrm{~nm}$ period gratings suspended on submicron thickness polyimide membranes [12]. A new type of grating for x-ray

2 The equation for de Broglie wavelength is $\lambda=\frac{h}{m v} \sqrt{1-\frac{v^{2}}{c^{2}}}$, where $h$ is Planck's constant $\left(6.626 \times 10^{-34} \mathrm{~J} \mathrm{~s}\right)$ and $m$ and $v$ are the particle's rest mass $(\mathrm{kg})$ and velocity $\left(\mathrm{m} \mathrm{s}^{-1}\right)$. 

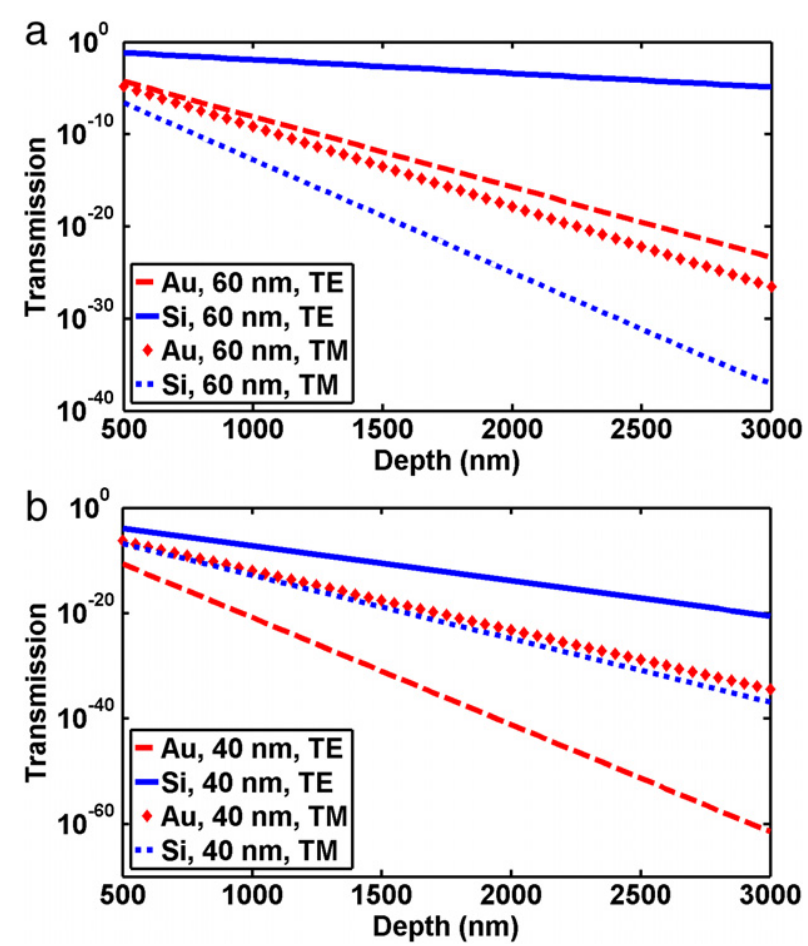

Figure 1. Comparison of simulated Lyman- $\alpha$ transmission through $2000 \mathrm{~nm}$ deep Si and Au gratings with $240 \mathrm{~nm}$ period and slits of $60 \mathrm{~nm}$ (a) or $40 \mathrm{~nm}$ (b). The polarization of the Au grating reverses if the slits are only $40 \mathrm{~nm}$ wide, and the Si grating approaches this point, as shown in (b).

diffraction [13] has provided impressive diffraction efficiency, but has insufficient open area to be ideal for most missions.

Most such applications to date have used gold gratings electroplated into pre-formed photoresist patterns $[9,12]$. The high conductivity of gold makes it very absorptive over most of the electromagnetic spectrum, including the deep UV and $\mathrm{x}$-ray. However, gold is structurally weak, requiring support for large-area gratings. Such support has been in the forms of the polyimide membranes used by Chandra (which close off the bottom of the grating, blocking particles) and the nickel support gratings used by MENA, which require additional processing steps.

This paper describes a path toward silicon UV filters with built-in bulk silicon supports. Such a structure is ideal since there are no thermal expansion mismatches between the grating and support, and no pressure need be applied to the thin grating to attach the support. Also, silicon has a Young's modulus close to that of stainless steel or nickel, so the back-side support structure is sufficient to support the gratings without additional processing. Since silicon is not as conductive as gold, the slit aspect ratio requirement is far greater than for a gold grating, but this requirement is met through innovative modifications to standard silicon processes. We first demonstrate through simulations the critical effects of material and geometry, as well as the performance of an ideal grating structure. We then fabricate and test a slightly simpler grating as a proof of principle, and finally close the loop between simulated and test data by explaining some interesting phenomena that arise under the assumption of 'perfect' gratings. A study of atomic layer deposition (ALD) coatings is currently underway. The work reported in this paper serves as a proof of principle, and is not targeted at filtering the Lyman- $\alpha$ line due to the lack of ALD coating in the current study.

\section{Simulation}

Simulations performed with GSOLVER, a professional Maxwell's equation solver from the Grating Solver Development Company ${ }^{3}$, were used to determine the target dimensions of the silicon gratings. Transmission of $121.6 \mathrm{~nm}$ photons through silicon gratings was simulated as a function of depth, slit width, and period. The transmission is dominated by slit width and depth, with grating periodicity being a secondary effect.

The material a grating is made of is probably the most important consideration, because the absorption is largely dependent on the material's complex index of refraction, most notably the imaginary (absorptive) component. Figure 1 shows the simulated transmission through gold and silicon gratings of both transverse electric (TE) mode light, with the electric field parallel to the grating lines, and transverse magnetic (TM) mode light, with the electric field perpendicular to the grating lines. Gratings with depth less than $500 \mathrm{~nm}$ would not attenuate the UV enough to be useful, and those deeper than $3000 \mathrm{~nm}$ would be prohibitively difficult to manufacture due to the extreme aspect ratio. The effect of the material is clearly evident. For gratings with $60 \mathrm{~nm}$ slits, a silicon (semiconductive) grating preferentially blocks TM mode light. While the gold (conductive) grating does the same, albeit less strongly, the inset shows that with $40 \mathrm{~nm}$ slits the gold grating preferentially blocks the TE mode by many orders of magnitude and the silicon grating still preferentially blocks the TM mode.

Conformal coatings of one or more material on silicon gratings enable minimization of the total transmission of a given wavelength, in this case Lyman- $\alpha$. Total transmission is defined here as the average of TE and TM modes. Strong dielectrics such as $\mathrm{SiO}_{2}$ and $\mathrm{Al}_{2} \mathrm{O}_{3}$ are very strongly absorbing in the far UV, and make ideal coatings for this purpose. It is also highly desirable to have a conductive layer coating the inside of the channels in order to avoid charging.

Since $\mathrm{SiO}_{2}, \mathrm{Al}_{2} \mathrm{O}_{3}$, and $\mathrm{Pt}$ can be conformally deposited even in high aspect ratio structures via ALD tools, simulations were run using thin layers on achievable grating geometries. Thick dielectric coatings can reverse the transmission of the two polarities rather than block both. For example, a $5 \mathrm{~nm}$ coating of $\mathrm{SiO}_{2}$ greatly reduces both TE and TM transmissions, but $20 \mathrm{~nm}$ blocks TE but lets TM through, the opposite of what would happen with no coating. Careful tailoring of material layers is critical to minimize total transmission.

The realities of plasma etching of high aspect ratio freestanding gratings impose further limitations on grating geometry. In particular, it is extremely difficult to etch 40 , 50, or $60 \mathrm{~nm}$ wide gratings that are $2000 \mathrm{~nm}$ deep. While the lithography technology is available to pattern to

\footnotetext{
3 http://www.gsolver.com.
} 


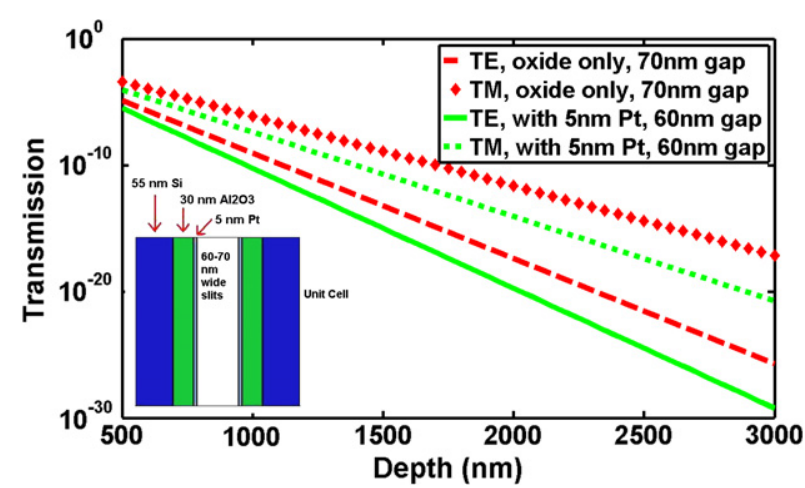

Figure 2. Simulated transmission of Lyman- $\alpha$ through a realistic layered grating structure: a silicon grating with period of $240 \mathrm{~nm}$ and etched slit width of $130 \mathrm{~nm}$ with ALD coating of $30 \mathrm{~nm}$ of $\mathrm{Al}_{2} \mathrm{O}_{3}$ on the sidewalls for a remaining slit width of $70 \mathrm{~nm}$. As an option, an additional $5 \mathrm{~nm}$ of ALD Pt coating narrows the slits to $60 \mathrm{~nm}$. The inset shows a top-view image of a unit cell of the grating.

such specifications, the diffusion limitation of plasma etch tools does not allow for such high aspect ratio etching without causing a bowing effect. A more realistic goal is to etch grating slits of $100-130 \mathrm{~nm}$ and use ALD coatings to narrow the slits to the desired range while also minimizing total transmission. Figure 2 shows the simulated Lyman- $\alpha$ transmission of such a grating with and without a thin $\mathrm{Pt}$ layer. With a $30 \mathrm{~nm}$ conformal aluminum oxide layer the total Lyman- $\alpha$ transmission is under $10^{-10}$ for a grating with $2000 \mathrm{~nm}$ depth. Addition of a $5 \mathrm{~nm}$ Pt layer drops the total transmission by another four to five orders of magnitude.

The above simulations demonstrate a tuning to minimize the transmission of a single wavelength, the Lyman- $\alpha$ band that dominates the solar UV flux. However, the gratings should also block other energetic radiation. The addition of multiple layers of material to a grating changes the absorption cross section across a wide spectrum. Figure 3 shows broadband transmission through the grating modeled in figure 2, including the $5 \mathrm{~nm}$ Pt coating. There are far more peaks and valleys present than in single-material grating simulations. This is only to be expected, and nearly the entire energetic UV spectrum transmits at under $10^{-5}$. While the UV is blocked, the visible and infrared portions of the spectrum pass through the grating almost unimpeded. This is considered a non-issue for two reasons: photons of those frequencies are not energetic enough to cause noise in a particle sensor, and in any case they will be strongly blocked by the microchannel plates typically used to enhance particle detection [14].

\section{Fabrication}

The fabrication of a freestanding, self-supported nanograting can be divided into four major parts: grating lithography, backside support lithography, etching, and optical tuning. The following provides a proof of principle of the first three pieces.

Both sides of silicon-on-insulator (SOI) chips are patterned in aluminum, first the front-side nanograting pattern, then the much coarser back-side support pattern. The buried oxide layer (BOX) of the SOI chips serves as an etch-stop for

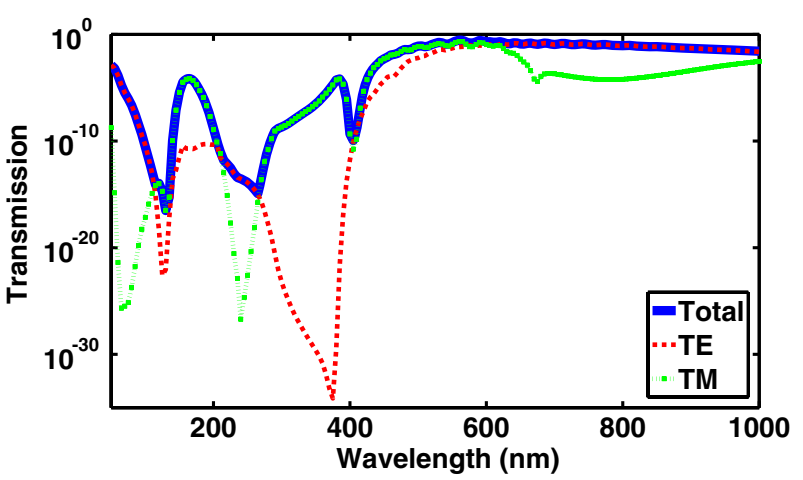

Figure 3. Simulated broadband transmission through a realistic layered grating structure. The blue line traces the sum of TE and TM transmission, which at this scale is essentially the higher of the TE and TM modes at any given wavelength.

the back-side etch, and is subsequently removed prior to the grating etch.

Nanoimprint lithography was chosen for patterning the gratings because of its advantages of speed, low cost, large pattern area (in this case $\sim 1$ inch $^{2}$ ), and high pattern density (120 nm half-pitch). Shadow-evaporation (evaporation at a sharp angle, such that each line is 'shadowed' by adjacent lines) of aluminum from both sides is used to protect the thermal-plastic resist lines during the plasma removal of resist at the bottom of the imprinted channels. Then, vertical evaporation of a $70 \mathrm{~nm}$ thick aluminum mask layer reverses the imprinted pattern and automatically plugs any pinhole flaws caused by imperfect mold separation in the lithography process. Liftoff of the resist and its protective metal in an acetone bath completes the grating lithography.

First a $240 \mathrm{~nm}$ period $1 \mathrm{D}$ grating with $50 \%$ duty cycle is deposited. Figure 4 shows SEM imagery of this portion of the process. Then an $11 \mu \mathrm{m}$ period anti-stiction grating with $300 \mathrm{~nm}$ wide lines is deposited at $90^{\circ}$ to the fine grating. After etching, this anti-stiction grating will enhance the rigidity of the long, thin lines of the fine grating. After depositing the metal mask, the device layer is spin coated with a protective layer of photoresist to allow back-side patterning. Both shadow-evaporation steps use high shadow angle $\left(\sim 75^{\circ}\right)$ to ensure that the bottom of each trench is left free. A schematic of the front lithography process is shown in figure 5 .

Back-side handle layer patterning is performed by standard photolithography and chemical etch of a $2 \mu \mathrm{m}$ layer of masking aluminum. The pattern is a $1 \mathrm{~mm}$ period $2 \mathrm{D}$ grating with $150 \mu \mathrm{m}$ support lines.

After patterning both sides of the chip, the chip is etched from the back side using an STS inductively coupled plasma (ICP) tool running an $\mathrm{SF}_{6} / \mathrm{C}_{4} \mathrm{~F}_{8}$ Bosch process that stops on the BOX layer. The BOX is removed by $\mathrm{C}_{2} \mathrm{~F}_{6}$ plasma in a LAM 9400 ICP tool, and the sample is removed from its carrier wafer by an acetone soak. An $\mathrm{O}_{2}$ plasma ash of the device layer removes resist residue from between the aluminum grating mask lines. Finally, a grating etch is performed on the front side in the STS. This is the most challenging task due to the requirements for high pattern density, high aspect ratio, and very smooth sidewalls. The process we 


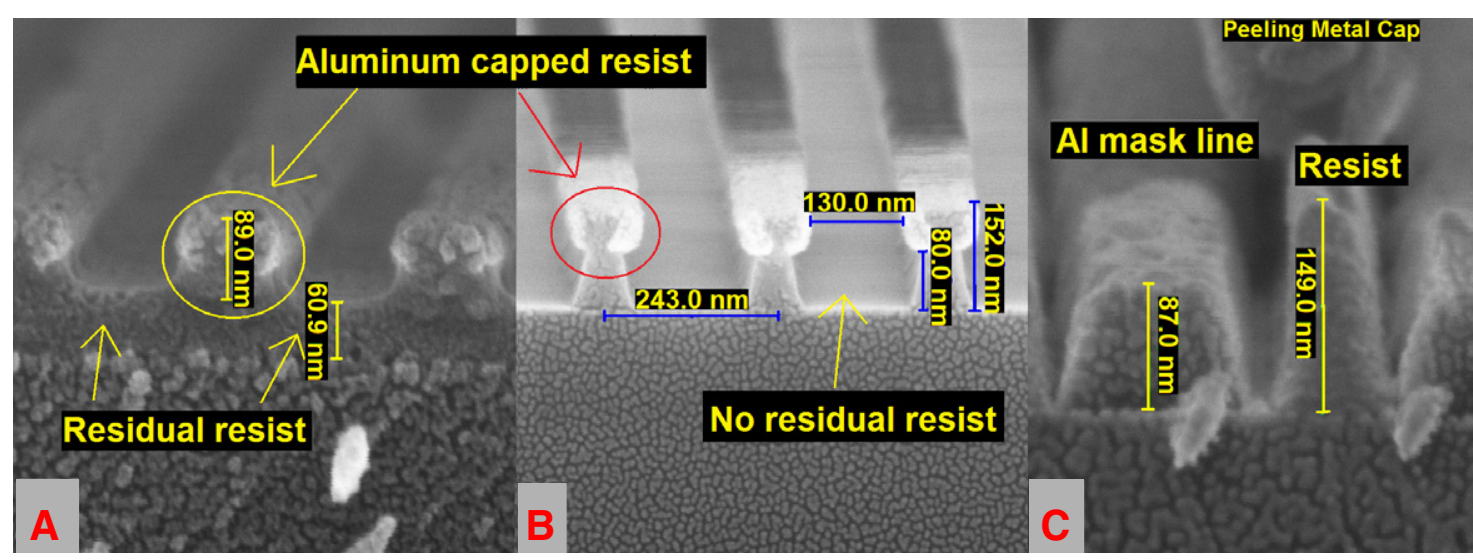

Figure 4. Cutaway SEM imagery of front-side grating lithography process. The edge-on shots are of the same sample after (A) imprint and dual shadow-evaporation of $5 \mathrm{~nm} \mathrm{Al,} \mathrm{(B)} \mathrm{residual} \mathrm{resist} \mathrm{etch,} \mathrm{and} \mathrm{(C)} \mathrm{direct} \mathrm{evaporation} \mathrm{of} 70 \mathrm{~nm} \mathrm{Al}$.

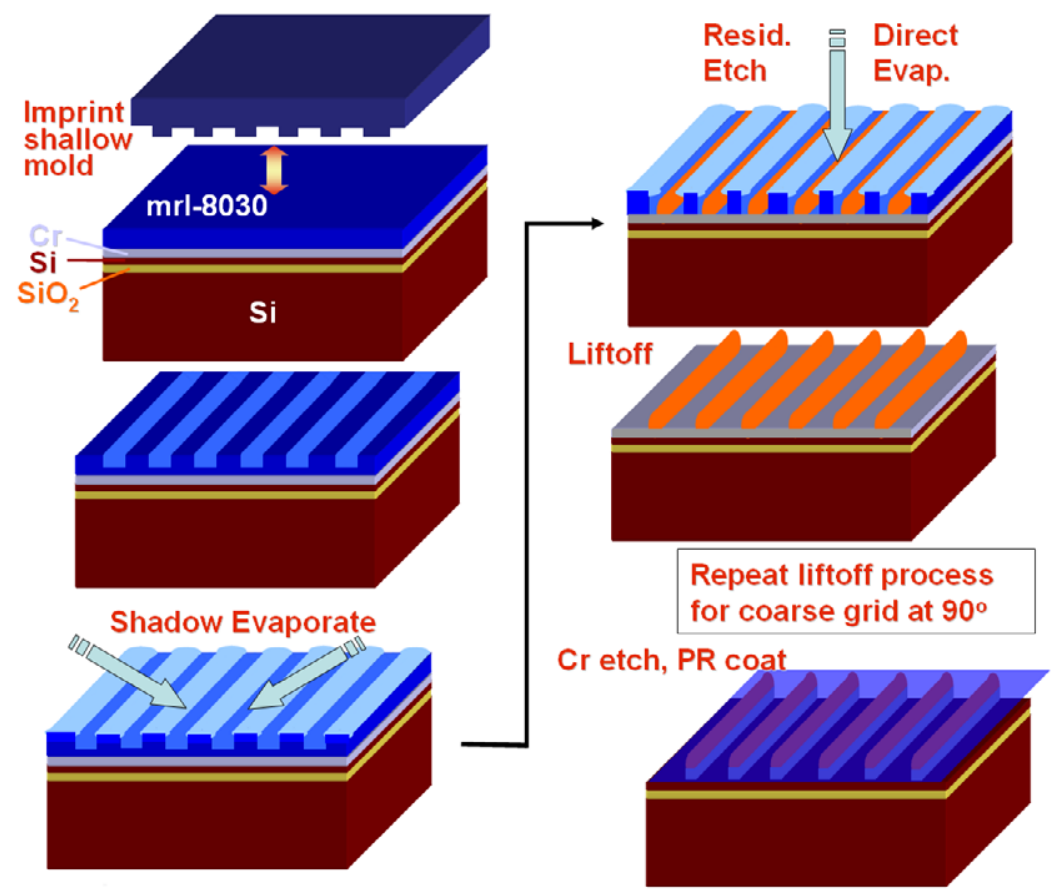

Figure 5. Grating lithography process. An SOI chip with mr-I 8030 thermal-plastic resist is imprinted with a $120 \mathrm{~nm}$ half-pitch mold and $5 \mathrm{~nm} \mathrm{Al}$ is shadow-evaporated from both sides. Next is residual layer removal, direct deposition of a $70 \mathrm{~nm} \mathrm{Al} \mathrm{hard} \mathrm{mask,} \mathrm{and} \mathrm{acetone} \mathrm{liftoff.}$ A second $11 \mu \mathrm{m}$ imprint at $90^{\circ}$ follows the same procedure. Then the surface is protected with photoresist during back-side processing.

demonstrated earlier [15] reduced the typical DRIE scalloping from $\sim 100 \mathrm{~nm} /$ cycle to $7 \mathrm{~nm}$ while reducing vertical etch rates to a controllable level. Subsequent modification of the etch chemistry, cycle times, and chamber pressure drastically reduced mask undercut and feature bowing. Ramping down the passivation time for each cycle helped straighten out the bottom portion of the grating slits. The dense plasma used by DRIE results in a significant amount of heat transfer to the substrate. The STS tool utilizes an electrostatic chuck with helium cooling of the back side of the wafer, but for suspended structures there is insufficient thermal path to the bulk. To enable additional cooling, the grating etch was broken into 2 min segments separated by $5 \mathrm{~min}$ of cool-down. Figure 6 shows the resultant freestanding, self-supported gratings.
The pattern shown in figure 6 is uniform across the entire grating area, which is a square of approximately $1.5 \mathrm{~cm}$. The dry etch tools do show an inhomogeneity in etch rate between the center of the chamber and the sides, but this potential problem is avoided by having a sacrificial BOX layer for the back-side etch and by using a thick photoresist layer on the carrier for the front-side etch. Even though the center of the pattern does etch through shortly before the edges, the additional etch/passivation steps do not noticeably change the grating morphology. The limit of pattern homogeneity appears to be the accuracy of the pattern transfer in the imprinting and pattern reversal steps, which is on the order of a few nanometers with a good mold. 

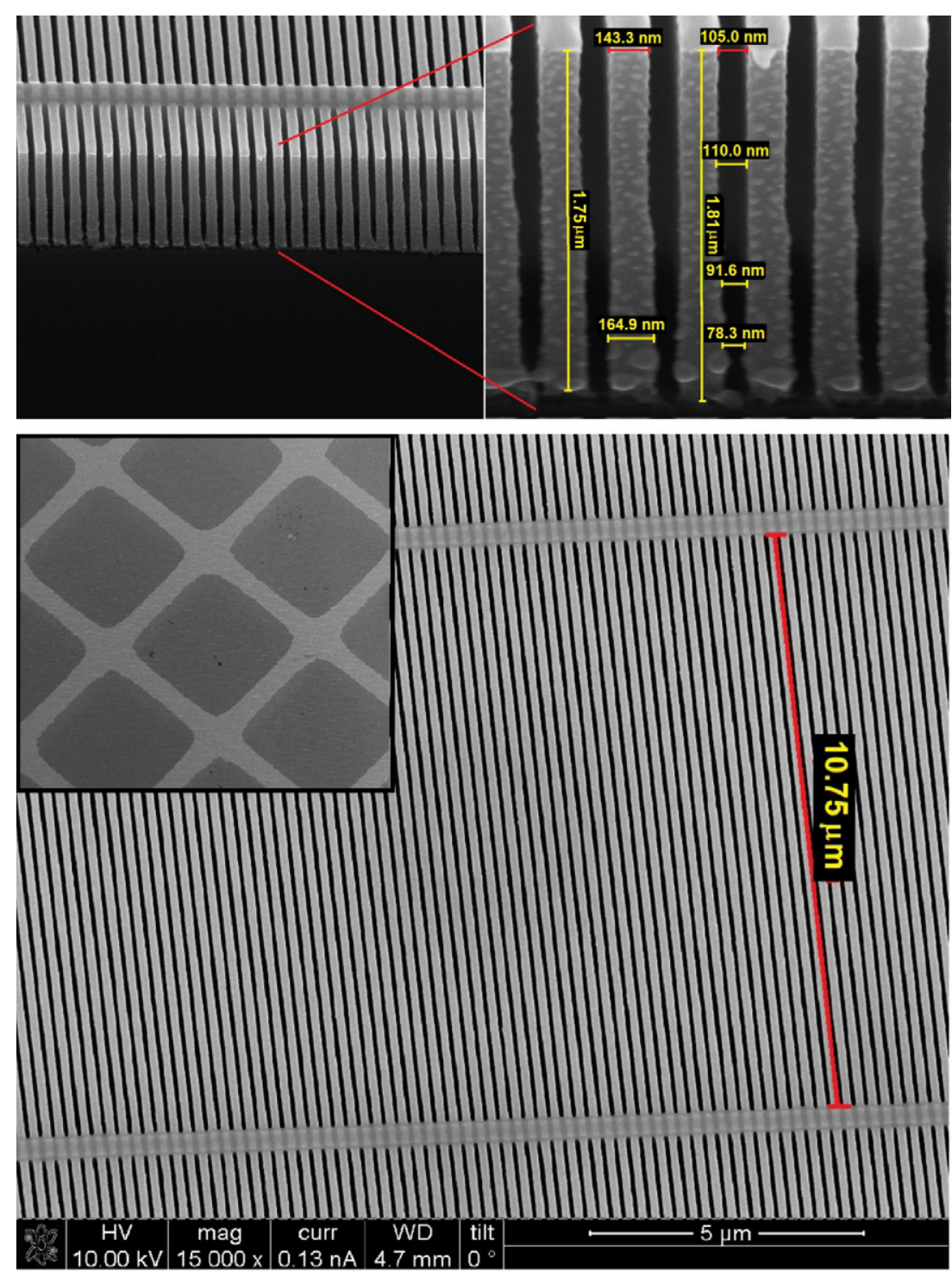

Figure 6. Freestanding grating structure. Top: the $240 \mathrm{~nm}$ pitch grating has a depth of $1.8 \mu \mathrm{m}$, and narrows as it goes downward due to multiple etch steps. Bottom: it is stabilized by an $11 \mu \mathrm{m}$ pitch grating at $90^{\circ}$. Inset: the whole structure is supported by a $500 \mu \mathrm{m}$ deep bulk silicon mesh of $150 \mu \mathrm{m}$ wide lines on a $1 \mathrm{~mm}$ pitch, seen at $65 \times$ magnification through the top grating.

\section{Optical testing}

Tests of the broadband optical transmission of the grating were performed with a xenon arc lamp for the UV (188-385 nm) and near IR $(775-870 \mathrm{~nm})$. The wide beam from the lamp was narrowed by an iris, then focused to a $\sim 3 \mathrm{~mm}$ point by a lens, and a second iris held the filter. A UV-vis capable optical fiber was placed $\sim 7 \mathrm{~mm}$ behind the filter to collect the transmitted light. The fiber led to a spectrometer with gratings having 600 blazes per millimeter and peak sensitivity at $400 \mathrm{~nm}$. The measurement integrated over $25 \mathrm{~ms}$ and averaged 100 spectra over a boxcar smoothing of 10 bins. A filter was used to flatten the UV portion of the lamp's emitted spectrum, but the visible portion saturated the spectrometer and was not usable, so a deuterium arc lamp was used to measure the visible portion of the spectrum $(400-800 \mathrm{~nm})$. This lamp setup used a microscope that collected light from a $\sim 900 \mu \mathrm{m}$ diameter spot and fed it into a spectrometer with peak sensitivity at $800 \mathrm{~nm}$. This measurement integrated over $15 \mathrm{~ms}$ with the same averaging and smoothing as the xenon lamp measurement. The resultant composite spectrum can be seen in figure 7. Also included is a spectrum simulated by GSOLVER using the fabricated dimensions seen in figure 6 .

\section{Discussion}

The measured transmission in figure 7 indicates that the UV is blocked with significant strength down to $195 \mathrm{~nm}$. However, there are some obvious anomalies between the measured and simulated data. In particular, there are sharp transmission dips at 250 and $430 \mathrm{~nm}$ in the simulated data, and the transmission from 500 to $900 \mathrm{~nm}$ is off by a factor of 100 . In-depth analysis proved that the discrepancies were all related to the simulation parameters, which model 'perfect' gratings, in other words gratings whose geometry and illumination can be exactly 


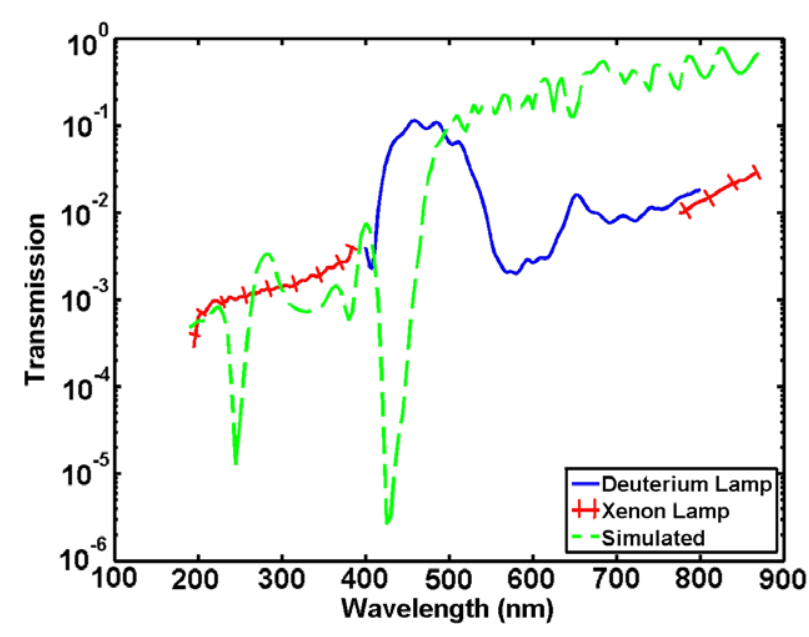

Figure 7. Broadband transmission through fabricated grating filter. The dotted line is a simulation of the grating geometry from figure 6 , and the other two lines are measured data.

modeled in GSOLVER or similar programs. In reality, there are effects due to the processing that cannot be fully modeled such as sidewall scalloping from DRIE, wearing away of the etch mask and possible redeposition of its material inside the grating, and pinhole flaws in the grating due to nonuniformities in the etch plasma. In addition, materials do not remain pristine during processing. They oxidize, become locally doped by plasma processing, retain residue from passivation, and so on, all of which can change their optical properties in potentially significant ways. These processing artifacts can have a major effect on the transmission characteristics of the grating. The data anomalies between the simulated and measured results will be addressed below.

The drop in measured transmission above $600 \mathrm{~nm}$ in figure 7 is partially caused by the residual aluminum mask lines on the surface of the grating structure. Addition of a $70 \mathrm{~nm}$ Al layer to the simulations resulted in a significant drop in simulated transmission around $800 \mathrm{~nm}$, bringing it into line with measured data. The difference between measured and simulated data at $600-800 \mathrm{~nm}$ is caused by geometric effects such as slight variations in the periodicity of the grating and sidewall roughness. Since the visible portion of the spectrum would be very strongly blocked in a real instrument by the measurement microchannel plates, this region is of less concern for space particle instrumentation than the anomalous simulated UV dropouts explained below.

The anomalous transmission dropouts in the simulated distribution have a variety of sources. To examine them in detail it is necessary to decouple the TE and TM modes. Figure 8 simulates the transmission of both modes and the total transmission through the grating. The first sharp drop in simulated TM transmission (location 1 in figure 8) is completely canceled out by the rise of the TE mode, and is also beyond the range of the measurement spectrometer. However, the TM drops at locations 2 and 3 are within the same region as the strong TE drop (location 4), and thus their absence from the measured data requires explanation.

Location 2 is at $250 \mathrm{~nm}$, which is very close to the period of the simulated grating. This transmission drop is

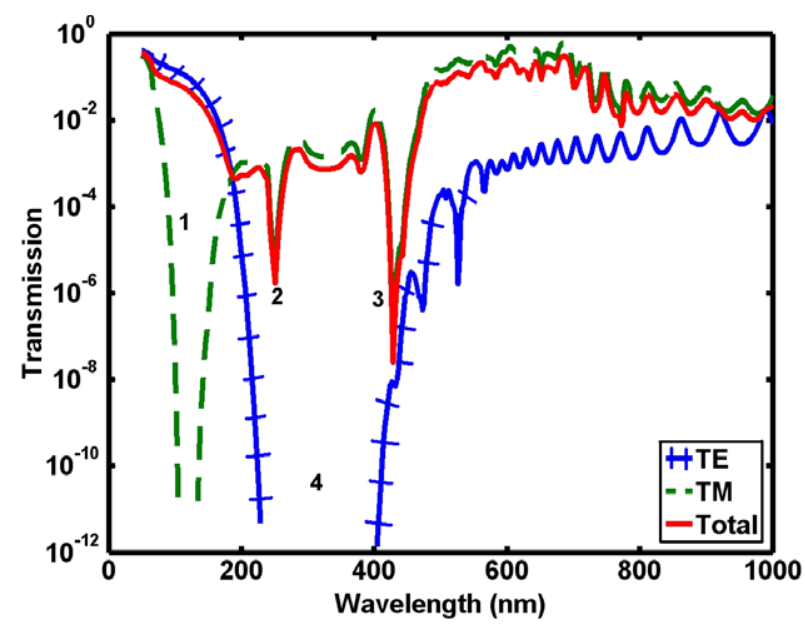

Figure 8. Simulated TE (crosshatched) and TM (dashed) broadband transmission through a fabricated grating filter. The peaks are labeled for subsequent reference. The solid red line is the total simulated transmission.

entirely dominated by the TM mode. It is caused by surface plasmon polaritons (SPPs), localized electromagnetic waves at a dielectric-metal interface caused by excitation near the entrance aperture of a slit grating [16]. These waves carry energy tangential to the surface, thus reducing transmitted intensity. These are normally only studied in metal gratings due to very sharp changes in transmission, but a semiconductor has enough free carriers to create a similar effect. Simulations varying the incoming light's incidence angle proved that the location 2 peak matched the expected location of the SPP dip, and the peak moved around based on the incidence angle. Since the light source used in the testing was not fully collimated, the SPP resonance and Wood's anomaly would not be in one specific set of wavelengths, and thus the angular variance of the incident light results in a smoother measured distribution.

The transmission dip at location 3 does not change at all due to variation of the incidence angle. This is because it is not caused by SPP or Wood's anomaly, since the wavelength is significantly larger than the grating period. However, this feature is very sensitive to slit width and the refractive index of the material, neither of which affect the SPP location much. The slit width can vary from slit to slit due to plasma nonuniformities during the etch process. In addition, there are small line dropouts scattered over the surface. These are not considered on the same level as the larger pinholes because they only affect a region of a few hundred nanometers, but are not uncommon and can smooth out this discrepancy region. The plasma process can also change the index of the material, primarily through doping effects. Changing the absorptive portion of the refractive index $(k)$ has a much larger effect than changing the real portion $(n)$. Simulations using different materials for the grating still had the SPP dip at $250 \mathrm{~nm}$, but the location of the number 3 dip varied significantly between gratings made of metal, semiconductor, and dielectric material.

The prior mentioned effects, detailed further in [17], serve to smooth out the transmitted spectrum such that the two 


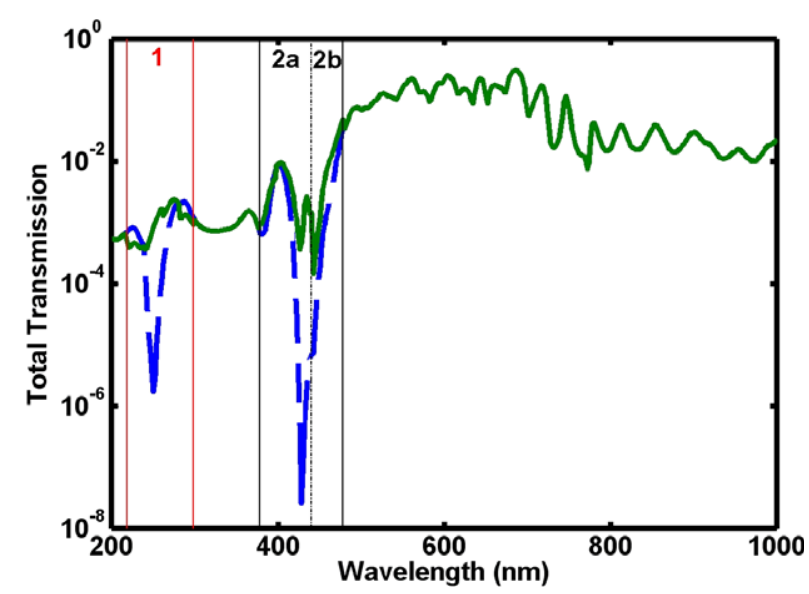

Figure 9. Aggregate effects of simulated variations in incidence angle, slit width, and refractive index on broadband transmission through a fabricated grating. The dashed blue line is the total transmission from figure 8 used as a reference. The solid green line in region 1 is averaged over light incident at $0^{\circ}, 5^{\circ}$, and $10^{\circ}$. In region $2 \mathrm{a}$ it is averaged over slits $\pm 10 \mathrm{~nm}$ from the default and refractive index variance, specifically reduction of $n$ by 0.25 , and reduction of $k$ by 0.15 . Region $2 \mathrm{~b}$ is the same as $2 \mathrm{a}$ without the $k$ reduction, since that would reduce $k$ below 0 .

spikes of interest disappear, as in the measured data. Figure 9 demonstrates this clearly by averaging over the following variations, with the addition of $70 \mathrm{~nm}$ aluminum mask material atop the lines.

- Incidence angle: normal, $\pm 5^{\circ}, \pm 10^{\circ}$.

- Slit width: baseline (as in figure 8 ), $\pm 10 \mathrm{~nm}$.

- Refractive index: single-crystal silicon, $k-0.15, n-0.25$.

Those variations were chosen simply to demonstrate the effects, but are definitely within the realm of possible variations within the tool and measurement apparatus.

One noteworthy design constraint discovered in the above analysis is that it is important to avoid building gratings with a period close to an integer multiple of the wavelength intended to be blocked. While creation of a grating with perfect geometry might allow strong blocking of the target wavelength by the SPP effect, the above-mentioned uncertainties in fabrication and measurement configuration make it equally likely that the target wavelength would land in the Wood's anomaly region of enhanced transmission.

This design space is further complicated by the fact that below $200 \mathrm{~nm}$ the SPP resonance for silicon is not determined solely by incidence angle and grating period, but also by effects unique to the material. While a change in incidence angle or period results in easily predicted resonances, the wide variation in $n_{\text {spp }}$ below $200 \mathrm{~nm}$ causes rapid changes in the SPP resonance wavelength that need to be taken into account. Coating of the grating sidewalls with an ALD metal layer would of course result in much sharper, predictable anomaly regions, and thus provide easier control of where the anomalies lie, but for a grating intended to block $121 \mathrm{~nm}$ light it would still be better to use a grating with a periodicity of, for example, 190 or $280 \mathrm{~nm}$ in order to better control the location of the second-order resonance peak (location 1 in figure 8).

\section{Conclusions and future work}

We have fabricated freestanding, self-supported, high aspect ratio nanogratings in bulk silicon. The slit widths can be further narrowed by an ALD layer of $\mathrm{SiO}_{2}$ or $\mathrm{Al}_{2} \mathrm{O}_{3}$ as a tuning step for targeting a specific minimum wavelength for the grating to reject. The oxide layer will have the added advantage of significantly increasing the absorption of energetic photons in the $50-150 \mathrm{~nm}$ wavelength band, and additional 2-10 nm ALD coating of a metal, probably platinum, might enable an additional advantage. Neutral atoms in transit through the slits will pass through the metal's surface electron cloud, possibly picking up an electron along the way. These ionized atoms can then be electrostatically guided away from the incoming particle stream for separate measurement. This would revolutionize neutral atom spectroscopy, since traditional methods involve far larger plates with grazing angle incidence. A three-year grant has been obtained through NASA to further explore these issues.

There are some important differences between doing UV filtering with gold gratings such as those used on MENA and using a silicon structure. The most important difference is that the silicon structure is self-supporting without need for further nickel supports or glued-on handles. The grating is a self-contained, self-supported chip with a wide handle region that can be used to attach it to an instrument. In addition, the silicon grating's strength results in a much wider open area than the gold grating, $20 \%$ compared to $10 \%$ even after application of the proposed ALD coatings, which should let a much larger number of particles through. Silicon has a much higher heat tolerance than gold. Conformally coated oxide and/or metal layers could experience thermal stress due to expansion coefficient mismatch at high temperatures, deforming the structure or delaminating the layers, but since the stress is experienced on both sides of grating lines it should cancel out.

In addition, the technique applied above can be used to create gratings with even higher aspect ratio if desired, resulting in stronger UV filtration. The gold technique was limited by the mechanical strength of the metal, and the grating lines became wavy if the depth was increased [9]. The variable depth of the silicon technique, the ability to add conformal layers to tailor the optical properties and aspect ratio, and the additional depth serving as a conversion surface for neutral atoms all make the silicon technique more adaptable than the gold technique. However, the electroplating used in the gold technique allows for plugging of fabrication holes with much more ease than the silicon technique. In the case of silicon, the liftoff process used for patterning the grating automatically plugs flaws caused by resist adhesion to the mold, but flaws caused by the plasma etching process are more difficult to address and could limit the maximum UV blocking capability of the grating. After ALD coating with a metal layer, it should be possible to use a similar technique to that used by the MIT group [9] to plug the pinholes, which uses the grating itself as a mask for standard photolithography and electroplating to plug the open areas. 


\section{Acknowledgments}

The authors would like to thank NASA for their support of this program under grant number NNX07AK29H. The authors would also like to thank the staff of the University of Michigan's Lurie Nanofabrication Facility for their assistance with tools and process parameters.

\section{References}

[1] Curdt W, Brekke P, Feldman U, Wilhelm K, Dwivedi B N, Schuhle U and Lemaire P 2001 The SUMER spectral atlas of solar-disk features Astron. Astrophys. 375 591-613

[2] Zurbuchen T, Bochsler P A and Scholze F 1995 Reflection of ultraviolet light at $121.6 \mathrm{~nm}$ from rough surfaces Opt. Eng. 34 1303-15

[3] Gronniger G, Barwick B, Batelaan H, Savas T, Pritchard D and Cronin A 2005 Electron diffraction from free-standing, metal-coated transmission gratings Appl. Phys. Lett. 87124104

[4] Gruntman M 1995 Extreme-ultraviolet radiation filtering by freestanding transmission gratings Appl. Opt. 34 5732-7

[5] Gruntman M 1997 Transmission grating filtering of 52-140 nm radiation Appl. Opt. 36 2203-5

[6] Balkey M M, Scime E E, Schattenburg M L and van Beek J 1998 Effects of gap width on vacuum-ultraviolet transmission through submicrometer-period, freestanding transmission gratings Appl. Opt. 37 5087-92

[7] Gruntman M 1997 Energetic neutral atom imaging of space plasmas Rev. Sci. Instrum. $\mathbf{6 8} 3617-56$
[8] Pollock C J et al 2000 Medium energy neutral atom (MENA) imager for the IMAGE mission Space Sci. Rev. 91 113-54

[9] van Beek J T M, Fleming R C, Hindle P S, Prentiss J D, Schattenburg M L and Ritzau S 1998 Nanoscale freestanding gratings for ultraviolet blocking filters J. Vac. Sci. Technol. B $163911-6$

[10] McMullin D R, Judge D L, Tarrio C, Vest R E and Hanser F 2004 Extreme-ultraviolet efficiency measurements of freestanding transmission gratings Appl. Opt. 43 3797-801

[11] McMorran B, Perreault J D, Savas T A and Cronin A 2006 Diffraction of $0.5 \mathrm{keV}$ electrons from free-standing transmission gratings Ultramicroscopy 106 356-64

[12] Canizares C R et al 2005 The Chandra high-energy transmission grating: design, fabrication, ground calibration, and 5 years in flight Publ. Astron. Soc. Pac. 117 1144-71

[13] Ahn M, Heilmann R K and Schattenburg M L 2007 Fabrication of ultrahigh aspect ratio freestanding gratings on silicon-on-insulator wafers J. Vac. Sci. Technol. B 25 2593-7

[14] Herrero F 1992 Light-trap design using multiple reflections and solid-angle attenuation-application to a spaceborne electron spectrometer Appl. Opt. 31 5331-40

[15] Mukherjee P, Kang M-G, Zurbuchen T H, Guo L J and Herrero F A 2007 Fabrication of high aspect ratio Si nanogratings with smooth sidewalls for a deep UV-blocking particle filter J. Vac. Sci. Technol. B $252645-8$

[16] Raether H 1988 Surface Plasmons on Smooth and Rough Surfaces and on Gratings (New York: Springer)

[17] Mukherjee P 2008 Modeling and technology development for sensitive near-solar particle measurements PhD Thesis University of Michigan, Ann Arbor, MI 